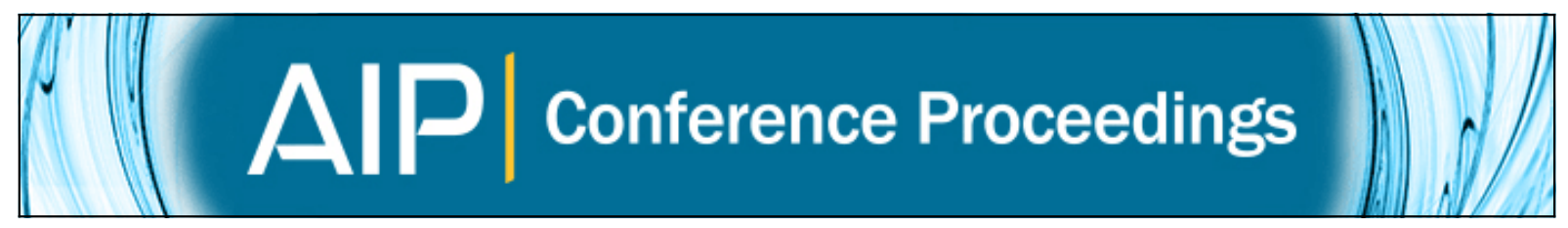

\title{
Modelling inhibition of AKT phosphorylation in acute myeloid leukemia
}

Y. A. Adi, F. Adi-Kusumo, L. Aryati, and M. S. Hardianti

Citation: AIP Conference Proceedings 1746, 020062 (2016); doi: 10.1063/1.4953987

View online: http://dx.doi.org/10.1063/1.4953987

View Table of Contents: http://scitation.aip.org/content/aip/proceeding/aipcp/1746?ver=pdfcov

Published by the AIP Publishing

Articles you may be interested in

A mathematical model of phosphorylation AKT in Acute Myeloid Leukemia

AIP Conf. Proc. 1723, 030001 (2016); 10.1063/1.4945059

Colour image segmentation using unsupervised clustering technique for acute leukemia images

AIP Conf. Proc. 1660, 090038 (2015); 10.1063/1.4915882

Parameter analysis for a mathematical model of the immune system in leukemia

AIP Conf. Proc. 1637, 1307 (2014); 10.1063/1.4907296

Periodicity in cell dynamics in some mathematical models for the treatment of leukemia

AIP Conf. Proc. 1493, 446 (2012); 10.1063/1.4765527

A delay differential equations mathematical model for the immune response in leukemia AIP Conf. Proc. 1493, 67 (2012); 10.1063/1.4765470 


\title{
Modelling Inhibition of AKT Phosphorylation in Acute Myeloid Leukemia
}

\author{
Y.A. Adi ${ }^{1,2, \text { a) }}$, F.Adi-Kusumo ${ }^{2}$, L. Aryati ${ }^{2}$, M.S. Hardianti ${ }^{3}$ \\ ${ }^{1)}$ Departement of Mathematics, Faculty of Mathematics \& Natural Sciences, University of Ahmad Dahlan \\ ${ }^{2)}$ Departement of Mathematics, Faculty of Mathematics \& Natural Sciences, University of Gadjah Mada \\ ${ }^{3)}$ Department of Internal Medicine, Faculty of Medicine, University of Gadjah Mada

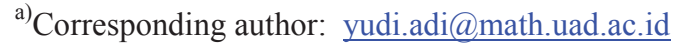

\begin{abstract}
Constitutive activation of PI3K/AKT signaling pathway has been observed in Acute Myeloid Leukemia (AML) that caused by the mutation of Fms-like Tyrosine Kinase 3 in internal tandem duplication (FLT3-ITD). Constitutive activation of AKT resulted in the regulation of apoptosis by the growth of abnormal cells that uncontrollably (AML blast). In our previous work we had consider a mathematical model of PI3K/AKT signaling pathways in phosphorylation AKT. In this paper we carry out a modification of the model by including synthesis and degradation of proteins as well as the effect of small molecule inhibitor of PI3K/AKT pathways. Perifosine is one of a small molecule inhibitor which has been widely known in the treatment of AML as AKT inhibitor. Our simulation result suggested that the administration of Perifosine may reduce the activity of AKT Phosphorylation. This result also support of the hypothesis that PI3K/AKT pathways is a potential target theraphy in AML.
\end{abstract}

Keywords : Acute Myeloid Leukemia, PI3K/ AKT, Small molecul Inhibitor, Modelling

\section{INTRODUCTION}

Acute Myeloid Leukemia (AML) is characterized by clonal expansion of early myeloid progenitor cell which some subtypes can be classified by distinct molecular and cytogenietic abnormalities [1]. Most cases of AML showed deregulated activity of the Phospoinositol-3-kinase (PI3K)/ protein kinase B (AKT)/mammalian target of rapamycin (mTOR) signal transduction. In the process of hematopoiesis regulation, PI3K, AKT, and mTOR are key component in a complex network of signaling proteins that promotes cell growth, proliferation, and survival. Overactivation of the PI3K/AKT/mTOR pathway has consistently been identified in AML which the most frequently identified transforming event are activating mutation in the receptor tyrosine kinase, Fms like tyrosine kinase 3 (FLT3) on the domain juxtamembrane internal tandem duplication (ITD) [2 - 5]. FLT3 activation promotes several downstream signaling pathways including $\mathrm{PI} 3 \mathrm{~K} / \mathrm{AKT} / \mathrm{mTOR}$. In normal conditions, low level activation of $\mathrm{PI} 3 \mathrm{~K} / \mathrm{AKT} / \mathrm{mTOR}$ pathways maintains cell survival but does not drive growth of cell division. PI3K activation is initiated through extracellular binding of ligands, which in turn triggers the activation of corresponding receptor tyrodine kinases (RTK) [4].

$\mathrm{PI} 3 \mathrm{~K}$ is a family of enzyme that have crucial rule in mechanism of cell regulation. The family of PI3K has been categorized into three classes, which class I PI3K is the best understood and are key players of multiple intracellular signaling networks that integrate a wide variety of signals and corelated with hematology malignancy. The most important PI3K substrate is phosphatidynositol 4,5 biphosphate (PtdIns(4,5)P2) (PIP2), which is phophorylated to yield phosphatidylinositol 3,4,5 triphosphate (PtdIns3,4,5P3) (PIP3). The most dominant downstreams effectors of PI3K are AKT, a sherine/threonine protein kinase contains an NH2 terminal pleckstrin homology $(\mathrm{PH})$ domain, which interacts with PIP3 and phospoinositide dependent kinase-1 (PDK1) at the plasma membrane resulting in AKT phosphorylation at threonine 308. For full activation, AKT also phosphorylation by mechanistic target of rapamycin complex 2 (mTORC2) at serine 473. In AML constitutive activation PI3K/AKT is a common feature, that is about 50 to $70 \%$ of patien with AML display phosphorilation of both Thr 308 and Ser 473 AKT.

Negative regulator of PI3K/AKT signal is a phosphatase and tensin homologue deleted on chromosome ten (PTEN). PTEN is a protein phosphatase that dephosphorylation the PIP3 back into PIP2. Dephosphorylation is essential to inhibit the signaling pathways of AKT. Loss of PTEN function results in the accumulation of PIP3 
and its downstream pathways. Another negative regulator is protein phosphatase 2A (PP2A) that can directly cause dephosphorylation at AKT. Dephosphorylation of AKT can also caused by PH domain leucine-rich repeat protein phosphatase (PHLPP) [6]. PHLPP can cause dephosphorylation of AKT phosphorylation on one site, which is Ser 473 or in both site site Thr 308 and Ser 473. In AML, the deregulation of the PI3K/AKT pathway is caused due to excessive activation of PI3K, PTEN deletion/ inactive, PP2A deletion/loss. These deregulation caused overactivation of AKT phophorylation that necessary for increased cell survival, proliferation, and leukemic transformation. Constitutive activation of AKT also phosphorylated and inhibit the transcription factor FOXO3a that caused its inactive [7]. FOXO3a is a transcription factor that plays an important role in the process of apoptosis and cell cycle regulation. Because of dysregulation of the PI3K/AKT signaling pathways has been observed in AML, this pathways and its downstreams effectors are considered to be promising targets for therapy.

Referring to our previous work [8], in this paper we modeled and simulated the AKT phosphorylation as a result of FLT3-ITD mutations that activate PI3K/AKT signaling pathways in cases of AML that carry out a modification of the previous model by including synthesis and degradation of proteins as well as the effect of small molecule inhibitor of PI3K/AKT pathways.

\section{TARGETING PI3K/AKT IN AML THERAPY}

Dysregulation of PI3K/AKT/mTOR pathway resulting from oncogenes amplification, oncogene activating mutations, inactivation of tumar suppresor genes, or upstream activation of RTK, has been demonstrated in a range of human malignamcies. In AML, this dysregulation commonly caused by the activating mutation of FLT3-ITD. There are a number of small molecule inhibitor of elements of this pathway in development, including PI3K inhibitor, AKT inhibitor, mTOR inhibitor, and dual mPI3K/mTOR inhibitor [1,3-5,7]. AKT is a key effector of PI3K/AKT pathways, but until now AKT inhibitor have been rarely tested in either preclinical nor clinical settings [5]. PI3K phosphorylates PIP2 generating PIP3 that in turn recruits proteins containing PH domains, such as AKT to the cell membrane. While in the cell membrane, AKT phosporylates at Thr308 and Ser473 for its full activation and generation of downstream pathways that promotes of cell prliferation and antiapoptotic signaling via several effectors. Downstream effects of this signal included inactivation of transcription factor GSK3 and FOXO3a through phosporylation that caused its retained in the cytoplasma and its transcriptional activity is prevented. AKT also contributes to chemotherapy resistance of AML blast [1]. Taken together, these fact provide a firm rationale for the development of PI3K/AKT inhibitor as therapies of AML.

To date, several small molecule inhibitors of PI3K/AKT pathway in AML have been developed, which demonstrated significant efficacy for reducing cell proliferation, inducing apoptosis and/or enhancing the efficacy of other therapies in various preclinical models of AML. The list of such inhibitors can be found at [1, 4,5,7]. Some AKT inhibitors obtained from that list are Perifosine, degeulin, triciribine, MK2206, GSK690693, and GSK2141795. Perifosine, the phospholipid analog, inhibits AKT by altering lipid rafts and preventing the membrane localization of AKT, resulting in apoptosis of AML cell lines and enhancement of etoposide cytotoxicity. Perifosine interact with AKT PH domain, preventing the membran recruitment and activation of AKT. The purine nucleoside analog, triciribin (TCN-PM) inhibits AKT phosporylation by interfering AKT PH domain and preventing its membrane localization. Degeulin is a nonspesific AKT inhibitor induced cell cycle arrest or apoptosis, and enhanced the sensitivity of AML cell lines or blast to etoposide or cytarabine while having relatively minimal efffects on normal CD $34^{+}$cells. Another strategy utilized to block AKT activation has been the development of small molecule inhibitors such as MK2206, GSK690693, and GSK2141795.

\section{MATHEMATICAL MODEL}

This mathematical model is a modification of the previous model with the additional presence of synthesis and degradation of proteins in the reaction. The representation of the biochemical reaction shown in Figure 1 below: 


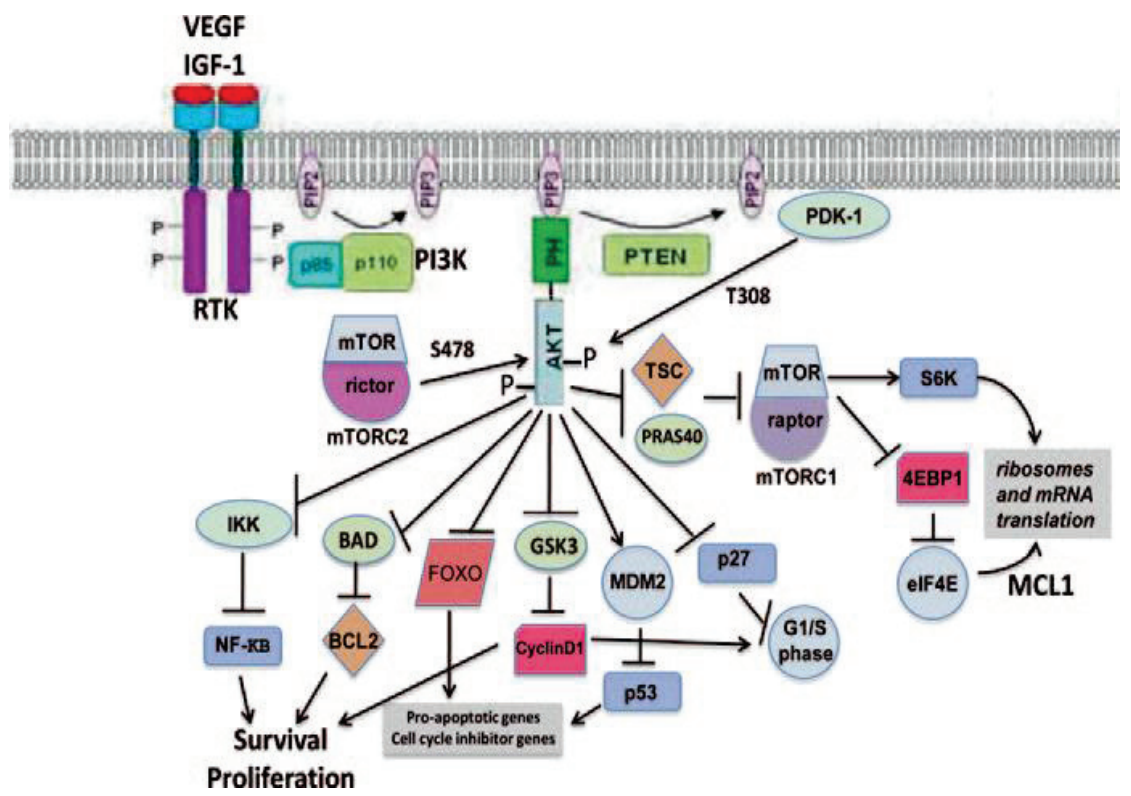

FIGURE 1. Schematic representation of the PI3K/AKT signaling module that drive oncogenesis in AML cells [5].

As shown in figure 1 , activation of PI3K by receptor stimulation results in the production of PtdIns $(3,4,5) \mathrm{P} 3$ at the plasma membrane. AKT subsequently translocates to the plasma membrane where it is phosphorylated by PDK1 and the mTORC2 complex at Thr308 and Ser473. On phosphorylation, AKT is released into the cytoplasm where it can both inhibitory phosphorylate multiple substrates, including TSC1/2 and/or PRAS40, FoxO transcription factors and GSK-3, and induce the activity of other substrates, such as mTOR as part of the mTORC1 complex. Negative regulators of the PI3K/AKT signaling module include PTEN, SHIP1, Ins(1,3,4,5)P4, PP2A, and PHLPP1/2. Here, we will modified our previous model of AKT phosphorylation that observed protein synthesis and degradation in the reactions and not observe a downsream pathways of AKT. A simplify schematic diagram of the PI3K/AKT signaling pathways to decsribed our mathematical model is shown in Figure 2.

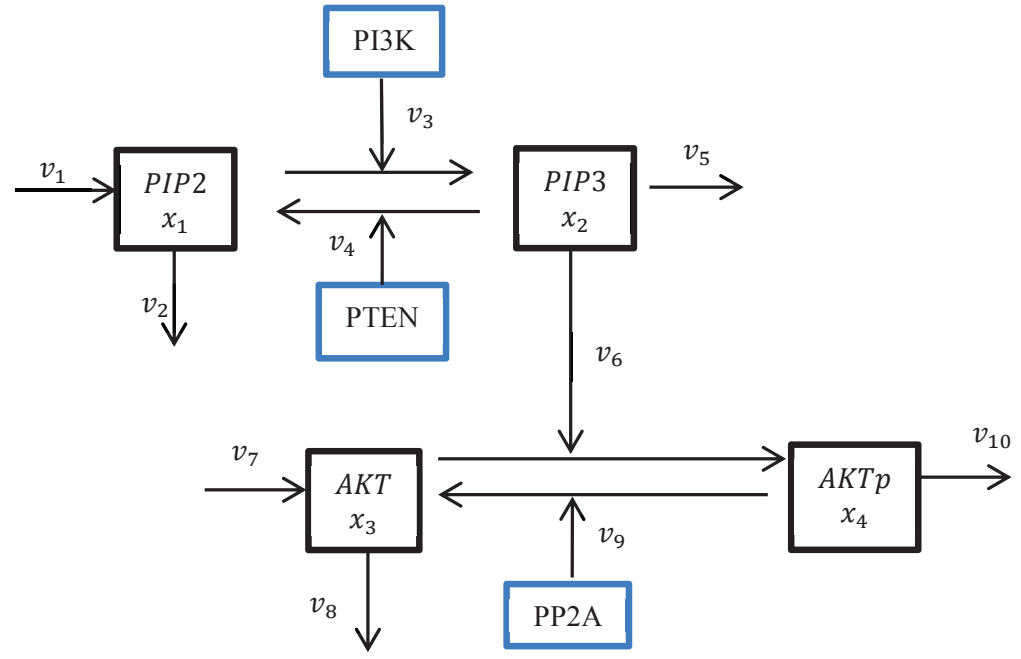

FIGURE 2. Schematic diagram of the biochemical reaction in PI3K/AKT signaling Pathway

From the schematic diagram in Figure 2, the chemical reaction can be divided in two levels of phosphorylation - dephosphorylation. The first level biochemical reactions is phosphorylation of PIP2 by PI3K as an enzyme to form PIP3 and dephosphorylation PIP3 by phospatase PTEN back to PIP2, and second level is 
phosphorylation AKT by PIP3 as enzyme to form AKTp and dephosphorylation of AKTpp by phospatase PP2A back to AKT (see [8] for detail derivation).

We denote the concentration of reactans : $x_{1}=[\mathrm{PIP} 2]=$ Concentration of PIP2, $x_{2}=$ Concentration of PIP3, $x_{3}=$ Concentration of AKT, $x_{4}=$ Concentration of AKTp. Then, modified the previous model of irreversible Michaelis Menten equation in [8], we have a mathematical model of PI3K/AKT signaling pathway is given by nonlinear ODE system (1) below:

$$
\begin{aligned}
& \frac{d x_{1}}{d t}=p_{1}-\frac{k_{1}[P I 3 K] x_{1}}{K_{1}+x_{1}}+\frac{k_{2}[P T E N] x_{2}}{K_{2}+x_{2}}-q_{1} x_{1} \\
& \frac{d x_{2}}{d t}=\frac{k_{1}[P I 3 K] x_{1}}{K_{1}+x_{1}}-\frac{k_{2}[P T E N] x_{2}}{K_{2}+x_{2}}-q_{2} x_{2} \\
& \frac{d x_{3}}{d t}=p_{2}-\frac{k_{3} x_{2} x_{3}}{K_{3}}-q_{3} x_{3}+\frac{k_{4}[P P 2 A] x_{4}}{K_{4}+x_{4}} \\
& \frac{d x_{6}}{d t}=\frac{k_{3} x_{2} x_{3}}{K_{3}}-\frac{k_{4}[P P 2 A] x_{4}}{K_{4}+x_{4}}-q_{4} x_{4} .
\end{aligned}
$$

\begin{tabular}{|c|c|c|c|c|c|}
\hline Parameter & Description & Unit & Range & Value used & References \\
\hline$p_{1}$ & $\begin{array}{l}\text { Synthesa/production of } \\
\text { PIP2 }\end{array}$ & Cell $\mathrm{mol}^{-1} \mathrm{~s}^{-1}$ & & 0.008 & 10 \\
\hline$q_{1}$ & Degradation of PIP2 & $s^{-1}$ & & 0.0013 & 9 \\
\hline$k_{1}$ & $\begin{array}{l}\text { Activation/phosphorylation } \\
\text { of AKT }\end{array}$ & $s^{-1}$ & & 0.2 & 8 \\
\hline$a_{1}$ & $\begin{array}{l}\text { Association of complex } \\
\text { PIP2:PI3K }\end{array}$ & Cell $\mathrm{mol}^{-1} \mathrm{~s}^{-1}$ & & $5 \times 10^{-6}$ & 8 \\
\hline$d_{1}$ & $\begin{array}{l}\text { Dissociation of complex } \\
\text { PIP2:PI3K }\end{array}$ & $s^{-1}$ & & 0.1 & 8 \\
\hline$a_{2}$ & $\begin{array}{l}\text { Association of complex } \\
\text { PIP3:PTEN }\end{array}$ & Cell $\mathrm{mol}^{-1} \mathrm{~s}^{-1}$ & & $5 \times 10^{-6}$ & 8 \\
\hline$d_{2}$ & $\begin{array}{l}\text { Dissociation of complex } \\
\text { PIP3:PTEN }\end{array}$ & $s^{-1}$ & & 0.1 & 8 \\
\hline$k_{2}$ & Deposphorylation of PIP3 & $s^{-1}$ & & 0.1 & 8 \\
\hline$k_{3}$ & Phosphorylation of AKTp & $s^{-1}$ & & 1 & 8 \\
\hline$a_{3}$ & $\begin{array}{l}\text { Association of complex } \\
\text { PIP3:PAKT }\end{array}$ & Cell mol $\mathrm{m}^{-1}$ & & $2.6 \times 10^{-4}$ & 8 \\
\hline$d_{3}$ & $\begin{array}{l}\text { Dissociation of complex } \\
\text { PIP3:AKT }\end{array}$ & $s^{-1}$ & & 0.1 & 8 \\
\hline$a_{4}$ & $\begin{array}{l}\text { Association of complex } \\
\text { AKTp:PP2A }\end{array}$ & Cell mol $\mathrm{s}^{-1}$ & & $1.7 \times 10^{-6}$ & 8 \\
\hline$d_{4}$ & $\begin{array}{l}\text { Dissociation of complex } \\
\text { AKTp:PP2A }\end{array}$ & $s^{-1}$ & & 0.1 & 8 \\
\hline$k_{4}$ & $\begin{array}{l}\text { Dephosphorylation of } \\
\text { AKTp }\end{array}$ & $s^{-1}$ & & 1.5 & 8 \\
\hline$p_{2}$ & $\begin{array}{l}\text { Production/synthesa of } \\
\text { AKT }\end{array}$ & Cell $\mathrm{mol}^{-1} \mathrm{~s}^{-1}$ & & 0.0015 & 9 \\
\hline$q_{2}$ & Degradation of PIP3 & $s^{-1}$ & & 0.02 & 9 \\
\hline$q_{3}$ & Degradation of AKT & $s^{-1}$ & & 0.0013 & 9 \\
\hline$q_{4}$ & Degradation of AKTp & $s^{-1}$ & & 0.00167 & 9 \\
\hline
\end{tabular}

where, $K_{1}=\frac{d_{1}+k_{1}}{a_{1}}, K_{2}=\frac{d_{2}+k_{2}}{a_{2}}, K_{3}=\frac{d_{3}+k_{3}}{a_{3}}, K_{4}=\frac{d_{4}+k_{4}}{a_{4}}$ are known as Michaelis-Menten constant. The set of kinetic rates $v_{i}$ and their kinetic rate constant are given in Table 1 .

TABLE 1. Kinetic rate constant 
We now simulate our mathematical model using the ode45 routine generating using Matlab. Parameters used on this simulation are depicted in Table 1 and the initial concentration of reactan we also refer to[8] given in Table 2 .

TABLE 2. Initial Concentration of the protein

\begin{tabular}{lll}
\hline Protein & $\begin{array}{l}\text { Concentration } \\
(\mathbf{n M})\end{array}$ & \multicolumn{1}{c}{ Range (nM) } \\
\hline PI3K & 10 & $10-100$ \\
PTEN & 270 & $0.1-350$ \\
PP2A & 150 & $4-150$ \\
PIP2 & 700 & $700-800$ \\
AKT & 700 & $10-900$ \\
\hline
\end{tabular}

Figure 3 shows the simulated protein activities used parameters on Table 1 and Table 2.

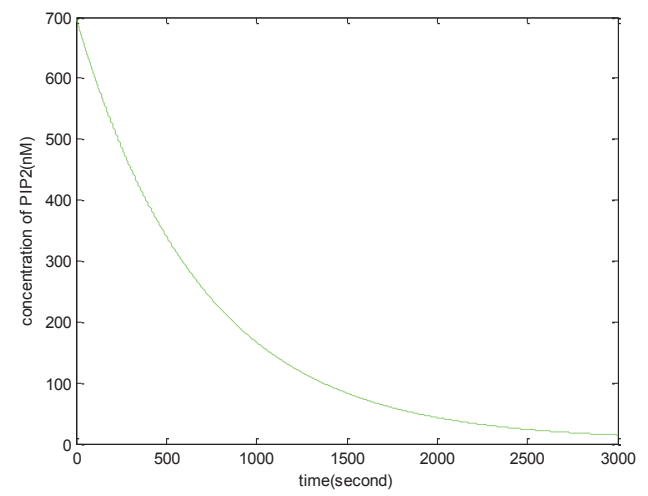

(a)

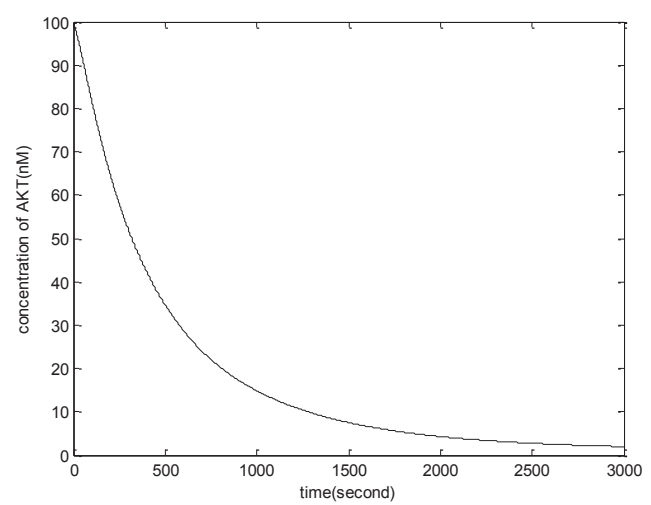

(c)

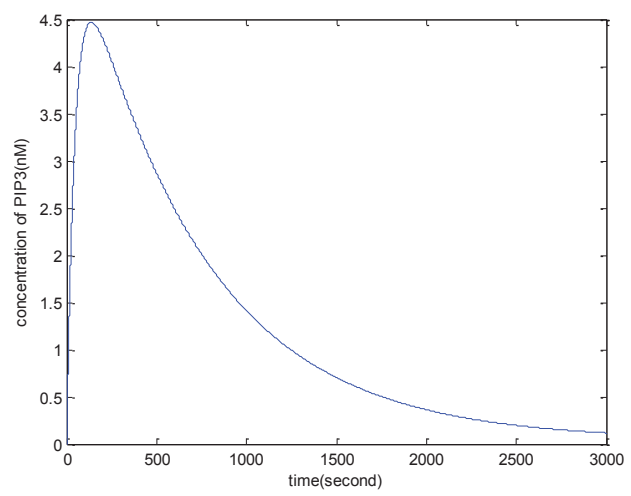

(b)

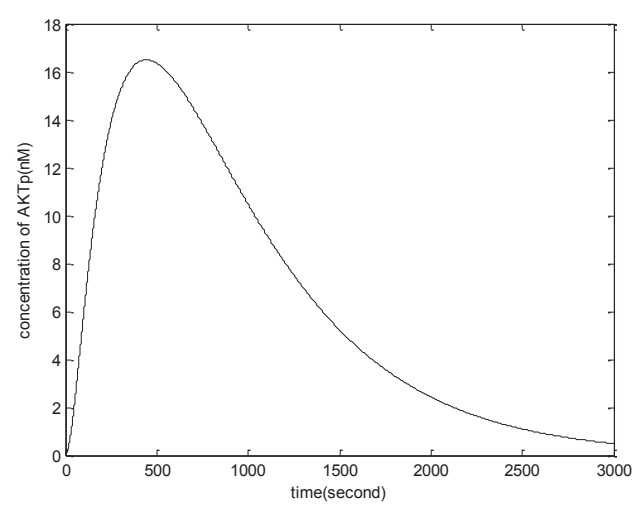

(d)

FIGURE 3. Simulated system dynamics of the protein activity.(a) Concentration of PIP2, (b). Concentration of PIP3, (c). Concentration of AKT, (d) Concentration of AKTp.

The initial condition is $[\mathrm{PIP} 2]=700 \mathrm{nM},[\mathrm{PI} 3 \mathrm{~K}]=100 \mathrm{nM},[\mathrm{PTEN}]=300 \mathrm{nM},[\mathrm{AKT}]=100 \mathrm{nM},[\mathrm{PP} 2 \mathrm{~A}]=$ $30 \mathrm{nM},[\mathrm{PIP} 3]=0,[\mathrm{AKTp}]=0$. In the initial conditbion of PI3K, we choose maximal concentration at $100 \mathrm{nM}$ due to increased PI3K activity in AML patiens [4]. The concentration of PIP2 convert to PIP3, so that the concentration of PIP3 increase and reach the maximum level within $\sim 150$ second. After reach thme maximal level, PIP3 will decline caused by degradation of PIP3. The level of PIP3 controlled the phosphorylation, AKTp, activation of AKT. AKTp reach its maximal level in about 500 second and will decline after the decrease of PIP3 level. 


\section{THE EFFECT OF AKT INHIBITOR}

PI3K/AKT pathways is a potential target for theraphy of AML patients. One of the most common small molecule inhibitor that inhibit AKT is Perifosine. Furthermore, we assume that Perifosine act as pure competitive inhibitor in the reaction of enzyme-inhibitor-substrate complex. With these assumption, as describes by Sauro in [11], if AKT concentration is increased, it posible for AKT to eventually out compete Perifosine, so that Perifosine alters the Michaelis constant $K_{3}$ but not the $k_{3}$. Therefore the equation for Perifosine inhibitor is shown in equation (2).

$$
\frac{k_{3} x_{2} x_{3}}{K_{3}\left(1+\frac{I}{K_{I}}\right)}
$$

with $I$ is the Perifosine and $K_{I}$ is the Perifosine binding dissociation constant. So if $I=0$, the equation reduces to $\frac{k_{3} x_{2} x_{3}}{K_{3}}$ as in equation (1). Now, use the equation (2) to equation (1), we have the simulation result of AKTp concentration shown in Figure 4.

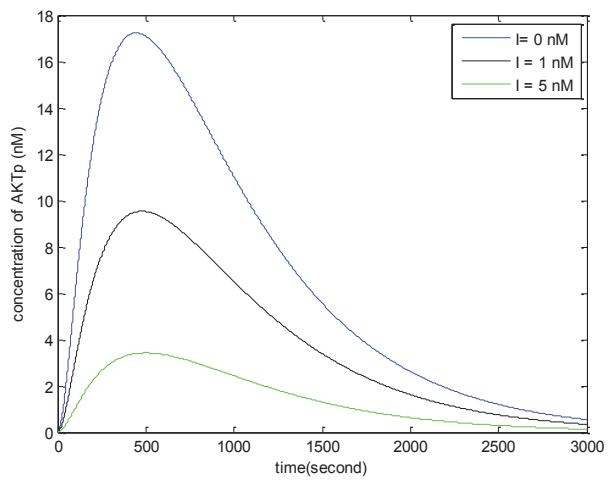

FIGURE 4. Concentration of AKTp with different concentration of Perifosine, $\mathrm{I}=0 \mathrm{~nm}$ (blue), $\mathrm{I}=1 \mathrm{nM}$ (Black), and $\mathrm{I}=5 \mathrm{nM}$ (green) with $K_{I}=1$.

Figure 4 gives simulated dynamcs of AKTp when different concentration of Perifosine is added into the system. The AKTp concentration is reduced proportionally when more concentration of Perifosine was added into the system.

\section{CONCLUSIONS AND FUTURE WORK}

In this work we carry out a modification of our previous model by including synthesis and degradation of proteins as well as the effect of small molecule inhibitor of PI3K/AKT pathways. We than consider Perifosine as AKT inhibitor that interact with AKT PH domain, preventing the membrane recruitment and activation of AKT. This model not consider protein substract in the downstream pathways of AKTp and crosstalk with another pathways. The administration of Perifosine as inhibitor of AKT in AML perhaps is an effective therapy such as simulation result in Figure 4. Mathematical analysis of this model is under preparation. Further studies required to determine the other target of small molecule inhibitor or multi targeted inhibitors so that the most effective combination to eradicate AML can be developed. In the future work we will also observe the other downstream pathways of AKTp, especially the FOXO3a trascription factor that play a critical rule in cellular apoptosis.

\section{REFERENCES}

[1] B.A. Carneiro, J.B. Kaplan, J.K. Altman, F.J. Giles, L.C. Platanias, Cancer Biology \& Therapy, 16(5), 648 - 656 (2015).

[2] C.H. Brandts, B. Sargin, M. Rode, et al, Cancer Res., 65(21), 9643 - 9650 (2005). 
[3] A.M. Martelli, M. Nyakern, G. Tabellini, R. Bortul, P.L. Tazzari, C. Evangelisti, L. Cocco, Leukemia, 20, $911-928$ (2006).

[4] L. Fransecky. L.H. Mochmann, C.D. Baldus, Molecullar and Cellular Therapies, 3(2), 1-17 (2015)

[5] B. Beagle and D.A. Fruman, The PI3K-AKT-mTOR Signaling Network in AML-Targeted Therapy of Acute Myeloid Leukemia, edited by Michael Andreeff (Springer Verlag, New York, 2015).

[6] H. Meng, Y. Jin, H. Liu, L. You, C. Yang, X. Yang, W. Qian, Journal of Hematology \& Oncology, 6(18), 1-14 (2013).

[7] R. Polak and Buitenhuis, Blood, 119(4), 911-923 (2012).

[8] Y.A. Adi, F.A. Kusumo, L.Aryati, M.S. Hardianti, A Mathematical Model of Phosphorylation AKT in Acute Myeloid Leukemia, AIP Conference Proceedings 1723, 030001 (2016).

[9] K.B. Wee and B. D. Aguda, Biophysics, 91, 857 - 865 (2006).

[10] P.J. Flaherty, A Kinetic Model for G Protein Coupled Signal Transduction in Macrophage Cells, Ph.D Desertation, University of California, Berkeley, 2006 see http:/www.eecs.berkeley.edu/Pubs/TechRpts/ 2007/EECS-2007-4.pdf

[11]H.M. Sauro, Enzyme Kinetics for Systems Biology (Ambrosius Pub, Washington, 2011) p.85 